\title{
Electroencephalography monitoring in the neonatal intensive care unit: a Chinese perspective
}

\author{
Zheng Wang ${ }^{1}$, Peng Zhang ${ }^{1}$, Wenhao Zhou ${ }^{1}$, Xiaoyu Zhou ${ }^{2}$, Yuan Shi ${ }^{3}$ Xiuyong Cheng ${ }^{4}$, Zhenlang Lin ${ }^{5}$, \\ Shiwen Xia ${ }^{6}$, Wei Zhou ${ }^{7}$, Guoqiang Cheng ${ }^{1}$ \\ ${ }^{1}$ Department of Neonatology, Children's Hospital of Fudan University, National Children's Medical Center, Shanghai, China; ${ }^{2}$ Department of \\ Neonatology, Children's Hospital of Nanjing Medical University, Nanjing China; ${ }^{3}$ Department of Neonatology, Children's Hospital of Chongqing \\ Medical University, Chongqing, China; ${ }^{4}$ Department of Neonatology, The First Affiliated Hospital of Zhengzhou University, Zhengzhou, China; \\ ${ }^{5}$ Department of Neonatology, Yuying Children's Hospital of Wenzhou Medical University, Wenzhou, China; ${ }^{6}$ Department of Neonatology, \\ Maternal and Child Hospital of Hubei Province, Wuhan, China; ${ }^{7}$ Department of Neonatology, Guangzhou Women and Children's Medical Center, \\ Guangzhou, China \\ Contributions: (I) Conception and design: G Cheng; (II) Administrative support: W Zhou, G Cheng; (III) Provision of study materials or patients: Z \\ Wang; (IV) Collection and assembly of data: Z Wang, P Zhang; (V) Data analysis and interpretation: Z Wang; (VI) Manuscript writing: All authors; \\ (VII) Final approval of manuscript: All authors. \\ Correspondence to: Guoqiang Cheng. Department of Neonatology, Children's Hospital of Fudan University, National Children's Medical Center, \\ Shanghai 200032, China. Email: gqcheng_cm@fudan.edu.cn.
}

Background: Electroencephalography (EEG) is an accessible technique for bedside monitoring of the cerebral function in the neonatal intensive care unit (NICU). The popularization of EEG in the field of newborns in China is relatively late compared with western countries. To learn more about current practices and improvement of EEG monitoring, we conducted a survey to describe current utilization of EEG in NICU in China.

Methods: A cross-sectional electronic survey with 21-items about EEG using in NICU was administered for pediatricians in China on the official website of "Questionnaire Star".

Results: A total of 251 participants were involved, in which 64\% of them reported using EEG. EEG was employed in NICUs of Children's hospitals (97\%), and grade III, class A hospitals (69\%). Besides, neonatal encephalopathy and suspected seizures were the most common indications for use. In clinical practice, the vast majority of physicians managed their patients on the basis of EEG (93\%). Pediatricians prefer to use conventional video-EEG (cEEG) to detect seizures and make the diagnosis of encephalopathy. Both amplitude integrated EEG (aEEG) (78\%) and cEEG (56\%) were mainly interpreted by neonatologists. However, only $56 \%$ of respondents had ever taken a formal EEG training course. Overall, $96 \%$ of the respondents reported that they would be interested in attending an education session on EEG in the NICU. aEEG interpretation was the most interesting part to learn (81\%). For those who were not using EEG, cost (43\%) and difficulty interpretation (30\%) were reported as barriers to use.

Conclusions: The utilization rate of EEG in NICU in China is significantly lower than the international level. There is an urgent need for standardized training and financial support for neonatologists in the use of EEG and interpretation of aEEG results.

Keywords: Electroencephalography (EEG); amplitude integrated EEG (aEEG); conventional EEG; neonatology

Submitted Oct 14, 2020. Accepted for publication Jan 17, 2021.

doi: $10.21037 / \mathrm{tp}-20-340$

View this article at: http://dx.doi.org/10.21037/tp-20-340 


\section{Introduction}

Advances in obstetric and neonatal care with more active management of very preterm infants (1) have increased survival in developed countries (2-4). However, there was no reduction in the incidence of neurological sequelae in these infants, who were at higher risk of brain damage during perinatal periods (5-7). Electroencephalography (EEG) can help detect seizures and subclinical central nervous system pathologies, inform treatment decisions, and correlates with neurodevelopmental outcomes in neonates at risk of neurological impairment (8).

Conventional video-EEG (cEEG) monitoring is the gold standard for assessing encephalopathy and detecting seizures in neonates (9). But in fact, that simultaneous recording of multichannel EEG in the neonatal intensive care unit (NICU) is difficult, and the interpretation of multichannel EEG can be problematic without a professional neurophysiologists with special expertise in neonatal EEG which are not available on a $24 \mathrm{~h}$ basis. The realization that EEG is useful not only for the detection of seizure, but also for the identification of babies suitable for neuroprotective therapies has led to an increase in demand for neonatal EEG (10). Therefore, amplitude integrated EEG (aEEG) has been widely used in NICU throughout the world as a more convenient and fast method for EEG monitoring (11). However, it does have relatively poor sensitivity for individual seizure detection, which may vary mainly due to the following factors: (I) The neonatologists' level of expertise in aEEG interpretation. (II) Shorter seizures, focal, multifocal or global seizures often go unnoticed using aEEG due to low time resolution and the position of the electrode in use. (III) Unnecessary or inadequate exposure to antiseizure medications for many infants. $(12,13)$. Therefore, to detect the seizure onsets, aEEG cannot be equivalently considered as EEG. For improved evaluation of electrocortical brain activity, the aEEG/EEG should be assessed together with repeated cEEGs or multi-channel EEG monitoring.

Compared with developed countries, the popularization of cEEG and aEEG in the field of newborns in China is relatively late and the establishment of the Neuro-intensive Care Alliance for neonates has not been completed until 2018. The Expert Consensus on the Clinical Application of Neonatal Amplitude-integrated EEG was first published in 2019 (14), and training courses on aEEG are gradually being developed and conducted. Since then, the EEG monitoring technology for critically ill newborns has gradually developed and matured in China. However, the process and results of studies of Chinese paediatricians using EEG have not been reported in details, and much remains unknown about their attitudes towards the tool. We conducted a nation-wide survey to characterize EEG usage in China and identify potential barriers to adoption of EEG technology among members of the Chinese Neonatologist Association.

We present the following article in accordance with the SURGE reporting checklist (available at http://dx.doi. org/10.21037/tp-20-340).

\section{Methods}

\section{Questionnaire design}

The questionnaire employed in this study was jointly designed by a team of neonatologists from the Children's Hospital of Fudan University between January and February 2019. It consists of 21 items and was divided into four dimensions, including socio-demographic characteristics of the respondents, current application of EEG monitoring in NICUs, interpretation and reasons for non-utilization of EEG (Appendix 1). The questionnaire was presented in the form of a Questionnaire Star (https://www.wjx.cn/, Hangzhou Oway Medical Technology, Changsha, China).

\section{Questionnaire affect test}

A preliminary experimental survey was performed in the Children's Hospital of Fudan University to evaluate the feasibility of the self-designed questionnaire. The researchers randomly assigned 30 pediatricians to take the test. It was found that the Cronbach's $\alpha$ coefficient was 0.971 , indicating a good reliability. The validity of the questionnaire was tested by factor analysis, and the KaiserMeyer-Olkin (KMO) value was 0.854 , which was suitable for factor analysis.

\section{Questionnaire distribution and collection}

The questionnaire was posted online through the Chinese Neonatologist Association website from March 12, 2019 to April 30, 2019, which reaches approximately 430 physicians. Questionnaires from the same hospital and incomplete responses were excluded from the final analysis. The answers to the questionnaire were grouped according to the type of hospitals: general hospital, maternity and infant 


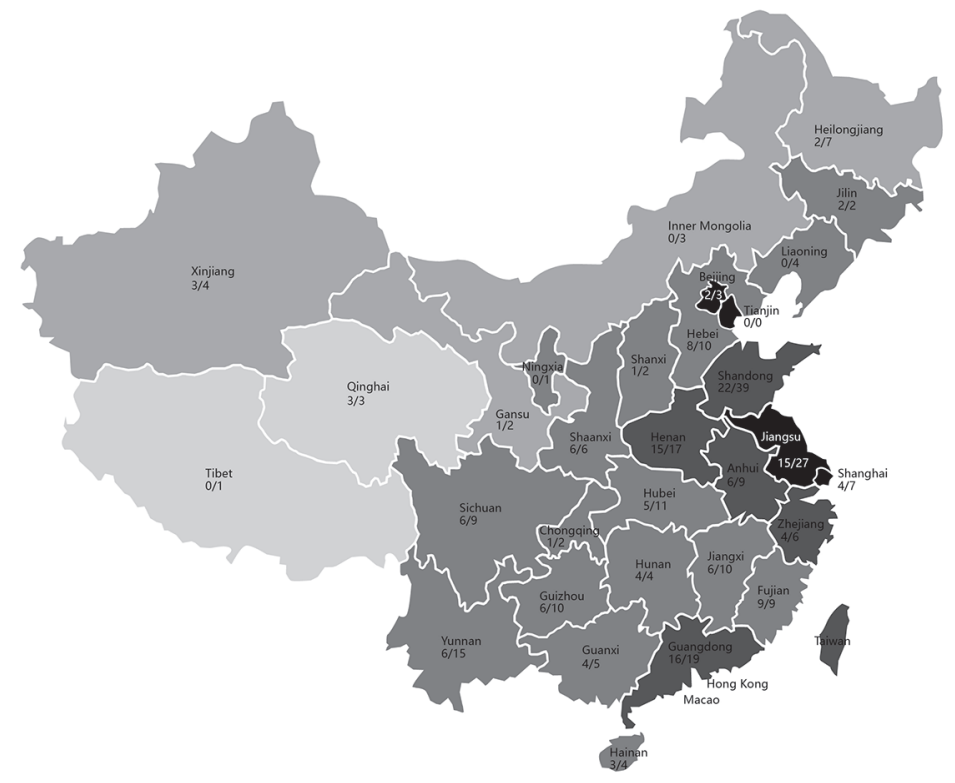

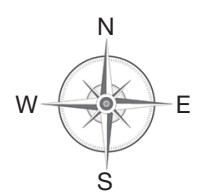
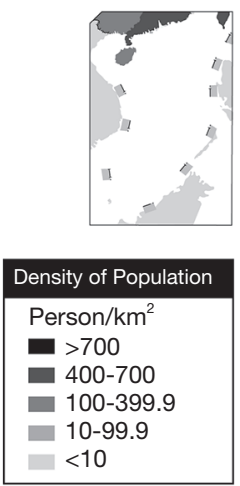

Figure 1 Geographic distribution of survey respondents. A grayscale map of China was divided into five levels depends on population density. The fractions represented the number of hospitals that participated in the survey (denominator) and the number of surveyed hospitals that provided EEG in the NICU (numerator) in each province. EEG, electroencephalography; NICU, neonatal intensive care unit.

hospital, or children's hospital.

\section{Informed consent}

All respondents participated voluntarily and anonymously, and informed that the data of the survey might be used for publication.

\section{Statistical analysis}

Statistics were analyzed using SPSS 23.0 (IBM Corp. Armonk, NY, USA). Bivariate analysis was conducted using chi-square or Fisher exact tests, when appropriate. Statistical significance was established using a $\mathrm{P}$ value of $<0.05$.

\section{Results}

\section{Characteristics of the survey respondents}

A total of 352 surveys were collected from neonatologists and pediatricians, representing an $82 \%(352 / 430)$ response rate. Thirty-two incomplete responses and 69 redundant responses from the same hospital were removed. Finally, 251 responses were included in the study, which represented 251 hospitals in 102 cities across 30 of the 34 provincial administrative regions in China (Figure 1).
The characteristics of the surveyed physicians and hospitals were presented in Table 1. Of the 251 respondents, 86\% (217/251) were Grade III and Class A hospitals which were the highest level hospitals in China, 59\% (149/251) were general hospitals, $27 \%(67 / 251)$ were maternity and infant hospitals, and 14\% (35/251) were children's hospitals.

\section{Application of EEG monitoring in NICUs}

\section{EEG utilization}

Of the 251 responding hospitals, 64\% (160/251) of them provided EEG in the NICU. The rate of children's hospitals (97\%) that provided EEG was significantly higher than that in maternity and infant hospitals $(72 \%)$ and general hospitals (52\%). Similarly, Grade III and Class A hospitals had a higher rate of EEG usage in comparison to Grade II and I hospitals (69\% vs. $29 \%, \mathrm{P}<0.001)$. The use of EEG was the highest in the NICU with more than 70 beds, whereas the rate of EEG usage was the lowest in the NICU with fewer than 20 beds ( $94 \%$ vs. $42 \%, \mathrm{P}<0.001$ ).

Of the hospitals that provided EEG, the use of aEEG $(60 \%)$ was significantly higher than that of cEEG (14\%) and aEEG combined with cEEG (26\%). Access to EEG monitoring modalities by profession and hospital types were shown in Table 2. The rate of cEEG usage in pediatricians 
Table 1 Characteristics of the survey respondents

\begin{tabular}{|c|c|}
\hline Variables & $N=251$ \\
\hline \multicolumn{2}{|l|}{ Profession } \\
\hline Neonatologist & $173(69 \%)$ \\
\hline Pediatrician & $78(31 \%)$ \\
\hline \multicolumn{2}{|l|}{ Professional title } \\
\hline Senior & $139(55 \%)$ \\
\hline Intermediate & $103(41 \%)$ \\
\hline Junior & $9(4 \%)$ \\
\hline \multicolumn{2}{|l|}{ Years of experience } \\
\hline $0-5$ & $4(2 \%)$ \\
\hline $6-10$ & $66(26 \%)$ \\
\hline $11-15$ & $62(25 \%)$ \\
\hline $16-20$ & $31(12 \%)$ \\
\hline$>21$ & $88(35 \%)$ \\
\hline \multicolumn{2}{|l|}{ Type of hospital } \\
\hline General hospital & $149(59 \%)$ \\
\hline Maternity and infant hospital & $67(27 \%)$ \\
\hline Children's hospital & $35(14 \%)$ \\
\hline \multicolumn{2}{|l|}{ Level of hospital } \\
\hline Grade III & $217(86 \%)$ \\
\hline Grade II \& I & $34(14 \%)$ \\
\hline \multicolumn{2}{|l|}{ NICU beds } \\
\hline$<20$ & $113(45 \%)$ \\
\hline $20-50$ & $92(37 \%)$ \\
\hline $51-70$ & $30(12 \%)$ \\
\hline$>70$ & $16(6 \%)$ \\
\hline
\end{tabular}

was significantly higher than that in neonatologists $(26 \%$ vs. $10 \%, \mathrm{P}=0.032)$. However, there were no significant differences in EEG modality among the three types of hospitals, including general hospital, maternity and infant hospital, and Children's hospital.

\section{Indications for EEG use}

The most common indicators for EEG monitoring in EEG-providing hospitals were encephalopathy and seizures. Overall, 23\% (37/160) of the respondents recognized that EEG monitoring was necessary for all hospitalized newborns in NICU.

In this study, the monitoring time of infants with encephalopathy and seizures has shown in Figure 2. Infants with encephalopathy were typically monitored for $2-5$ hours (44\%). Sixty-eight percent of the respondents reported that they monitored the patients' brain for more than 6 hours for infants with seizures. There was no significant difference in the time of EEG monitoring for infants with encephalopathy and seizures among the three different types of hospitals.

\section{Interpretation of EEG}

The 138 hospitals that provided aEEG clinical monitoring results showed that the interpretation of the obtained results was mainly performed by neonatologists (78\%) and pediatric neurologists (22\%). cEEG interpretation was performed by neonatologists $(56 \%)$ and pediatric neurologists (44\%) in 64 hospitals that provided cEEG. In addition, only $56 \%$ of respondents reported receiving a hospital-run training course on how to read and interpret EEG, while $63 \%$ said they had received no training and

NICU, neonatal intensive care unit.

Table 2 EEG usage by profession and hospital type

\begin{tabular}{|c|c|c|c|c|c|c|c|}
\hline EEG modality & \multicolumn{3}{|c|}{ Profession } & \multicolumn{4}{|c|}{ Hospital type } \\
\hline Only cEEG & 13 (10\%) & $9(26 \%)$ & 0.032 & $10(13 \%)$ & 7 (15\%) & $5(15 \%)$ & 0.946 \\
\hline
\end{tabular}

aEEG, amplitude-integrated electroencephalography; cEEG, conventional electroencephalography. 


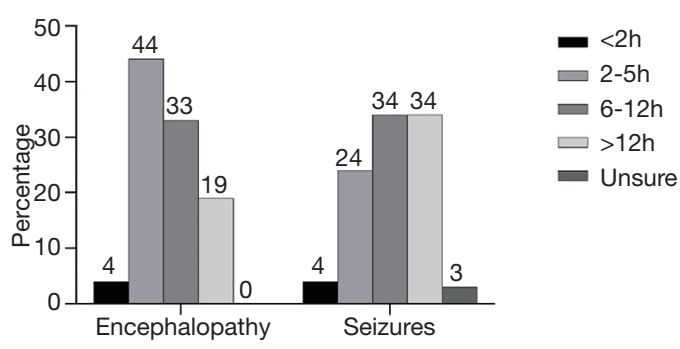

Figure 2 EEG monitoring time for infants with encephalopathy and seizures. EEG, electroencephalography.

being taught by themselves.

As can be seen from Table 3, the rate of interpretation of aEEG by neonatologists from maternity and infant hospital was significantly higher than that of children's hospitals $(\mathrm{P}=0.014)$, while the rate of interpretation of cEEG by pediatric neurologists was significantly lower than that of general hospitals $(\mathrm{P}=0.001)$ and children's hospital $(\mathrm{P}<0.001)$.

\section{Reasons for Non-utilization of EEG}

Thrity-five percent (87/251) of responding hospitals indicated that they do not use EEEG in their NICUs. Several factors were identified as reasons for not offering EEG, including high cost for EEG devices (43\%), difficult interpretation (30\%), available in other department (20\%), and concerns about the complicated operation of EEG (8\%).

From Table 4, it was clear that the high cost of EEG devices and difficult in EEG interpretation were the main reasons for the absence of EEG in general hospitals and maternity and infant hospitals, respectively.

\section{Discussion}

This study is the first national assessment of the current utilization of EEG monitoring in the department of NICUs in China. The respondents were mainly neonatologists with abundant clinical experiences who worked in grade III and class A hospitals. Therefore, to a certain extent, findings of this study could objectively reflect the current development of EEG monitoring in the field of newborns in China.

In a multinational survey, EEG was used in more than $90 \%$ of NICUs $(15,16)$. However, in this study, only $64 \%$ of the responding hospitals in China used EEG for continuous monitoring of newborn patients. Moreover, different from developed countries, the high cost of devices has become a major obstacle to the clinical implementation of EEG projects in China $(15,16)$. In addition, nearly onethird of hospitals did not provide EEG because of the difficulty in interpretation, which may be related to the lack of awareness of multidisciplinary team cooperation among pediatricians in China and the lack of standardized training on EEG. Therefore, there is an urgent need for more EEG training courses to improve EEG operation and interpretation ability.

Consistent with the findings of previous studies, the utilization rate of EEG monitoring in children's hospitals, grade III and class A hospitals was higher, while that in general hospitals and small-scale hospitals was relatively lower $(17,18)$. As more critically ill newborns are admitted to children's hospitals and grade III, class A hospitals, children are at higher risk of brain injury, it is necessary for pediatricians to carry out continuous cerebral monitoring in the diagnosis and treatment process.

Our results indicated that practitioners from the two specialties tend to differ in their preferred patterns: for most clinical scenarios, the majority of neurologists prefer cEEG, while neonatologists were more likely to adopt aEEG. These results are similar to previous surveys of EEG use in the NICU (15-17). Each subspecialty tends to its own monitoring modality, which may be due to familiarity, accessibility, timeliness of EEG analysis, or perceived confidence in interpretation. Neonatologists are satisfied with current practices, but may not be aware of the limitations of aEEG and the additional diagnostic clarification that cEEG can provide. cEEG was perceived as more accessible to neurologists than neonatologists, which may be due to the close relationship neurologists have with neurophysiology services and technicians. Overall, the discrepancies in the availability and accessibility of longterm EEG monitoring between specialties highlight the need for effective collaboration among subspecialties to improve patient care.

In this study, the vast majority of respondents believe that EEG was an important means to predict or judge whether infants had encephalopathy or suspected seizures. This suggests a potential gap in monitoring other high-risk groups of neonates such as central nervous system (CNS) infection, CNS trauma, inborn errors of metabolism, and premature infants with severe intraventricular hemorrhage. Recent studies have demonstrated that aEEG can be used to continuously monitor brain function in infants with inborn errors of metabolism (19) and premature infants with posthemorrhagic hydrocephalus (20-22), suggesting 
Table 3 aEEG and cEEG interpretation among the three types of hospitals

\begin{tabular}{lccc}
\hline EEG interpretation & General hospital, $n=68$ & Maternity and infant hospital, $n=48$ & Children's hospital, $n=34$ \\
\hline aEEG & $56(72 \%)$ & & \\
Neonatologist & $22(28 \%)$ & $43(90 \%)$ & $23(68 \%)$ \\
Pediatric neurologist & $5(10 \%)$ & $11(32 \%)$ \\
CEEG & $41(53 \%)$ & $39(81 \%)$ & $10(29 \%)$ \\
Neonatologist & $37(47 \%)$ & $9(19 \%)$ & $24(71 \%)$ \\
Pediatric neurologist & & & $<0.001$ \\
\hline
\end{tabular}

aEEG, amplitude-integrated electroencephalography; cEEG, conventional electroencephalography.

Table 4 Rationale for not using EEG

\begin{tabular}{lccc}
\hline Reason & General hospital, $\mathrm{n}=68$ & Maternity and infant hospital, $\mathrm{n}=18$ & Children's hospital, $\mathrm{n}=1$ \\
\hline Too expensive & $30(44 \%)$ & $6(33 \%)$ & $1(100 \%)$ \\
Difficult to interpret & $18(26 \%)$ & $8(44 \%)$ & $0(0 \%)$ \\
Another department has EEG capability & $14(21 \%)$ & $3(17 \%)$ & $0(0 \%)$ \\
Other & $6(9 \%)$ & $1(6 \%)$ & $0(0 \%)$ \\
\hline
\end{tabular}

that aEEG has potential for broader use than is currently recognized.

The American Clinical Neurophysiology Society recommends that infants at-risk for seizures need extended monitoring because "a 1-hour EEG is considered inadequate to screen for seizures" (9). Abnormalities of the neonatal EEG/sleep cycle may be more reliably to be detected over long periods ( 3 to 4 hours) or in serial recordings. An expandable study conducted in pediatric ICUs found that EEG monitoring for 24 hours or longer significantly increased sensitivity to screening for seizures, thereby altering and improving clinical management practices in $59 \%$ of patients (23). However, it is unclear whether all of these neonates at risk of seizures require more than 24 hours of EEG monitoring. In this study, more than half of the responding hospitals conducted clinical EEG monitoring of infants with brain injury and seizures for more than 6 hours. The monitoring time of our survey is not as long as that of published surveys, probably because China has a high population density and a relatively large number of patients who needed EEG monitoring. This resulted in the monitoring time of some neonates with electrographic seizures have to shorten or not to monitor, generating the omission of the treatment of patients. Therefore, it is urgent for hospitals to purchase more EEG devices to meet the needs and improve the scalp care during EEG monitoring in China.

Neonatal EEG is generally considered to be the most difficult type of EEG to interpret because of the variety of age. In clinical practice, although a brief EEG record can be obtained by using other devices, results may be interpreted hours or even days later, which is difficult to meet the clinical needs of infants with epilepsy or other diseases to correctly choose the next step of treatment. As a result, neonatologists had to make up for the study's shortcomings by conducting in-depth studies, even though they had no formal training in EEG training in EEG recording and interpretation (15). This situation also exists in China and even worsen. Our survey found that the rate of interpretation of both aEEG and cEEG by neonatologists was higher than that by pediatric neurologists. Although the majority of the respondents have more than 10 years clinical experience, whereas only $56 \%$ had attended a formal training on EEG interpretation and $96 \%$ indicated they would be interested in attending an education session on neonatal EEG especially on aEEG interpretation (81\%). This indicated that the ability to interpret results of EEG is at a low level among neonatal staff in China. Therefore, formal training courses on aEEG interpretation need to be available for pediatrics to improve their confidence 
and accuracy of aEEG interpretation. It also perhaps can broaden aEEG utilization and acceptance.

The strength of this study is that it included three different types of hospitals located across 30 provinces and districts in China. Through the analysis of the questionnaire results, it's helpful to objectively understand the current status of EEG monitoring in NICUs, identifying obstacles and aspects for improvement in the implementation of EEG monitoring in China.

There are several limitations in this study. Although the survey covered nearly $90 \%$ of provinces in China, more than 30,000 hospitals had registered in the domestic. The negative response bias (nonresponse error) may limit the generalizability of the findings. Most units reported that they had a high level of NICU monitoring and a matured team of experienced staff. Therefore, the rate of EEG may be higher than the actual situation. This also should be reflected in interpreting the results, as they may actually be an overestimate of the general population. In addition, this study has self-report bias due to the inherent defects of questionnaire survey. Our study may have selection bias because majority of respondents were neonatologists, more pediatric neurologists should be involved to further study.

In conclusion, EEG is an important bedside-monitoring method to evaluate the cerebral function of critically ill newborns. Currently, EEG technology has been gradually implemented in many hospitals in China, but it is not common. There is significant heterogeneity in most aspects of EEG practices. Barriers to expanded use include high cost of devices, the difficulties of explaining EEG, and insufficient investment in the education and training of professional pediatricians. By increasing funding, strengthening multidisciplinary teams, and standardizing EEG operation and interpretation skills, Chinese pediatricians may be able to effectively improve the management of the infants at-risk for seizures.

\section{Acknowledgments}

Funding: None.

\section{Footnote}

Reporting Checklist: The authors have completed the SURGE reporting checklist. Available at http://dx.doi. org/10.21037/tp-20-340

Data Sharing Statement: Available at http://dx.doi. org/10.21037/tp-20-340

Conflicts of Interest: All authors have completed the ICMJE uniform disclosure form (available at http://dx.doi. org/10.21037/tp-20-340). The authors have no conflicts of interest to declare.

Ethical Statement: The authors are accountable for all aspects of the work in ensuring that questions related to the accuracy or integrity of any part of the work are appropriately investigated and resolved. All respondents participated voluntarily and anonymously, and informed that the data of the survey might be used for publication.

Open Access Statement: This is an Open Access article distributed in accordance with the Creative Commons Attribution-NonCommercial-NoDerivs 4.0 International License (CC BY-NC-ND 4.0), which permits the noncommercial replication and distribution of the article with the strict proviso that no changes or edits are made and the original work is properly cited (including links to both the formal publication through the relevant DOI and the license). See: https://creativecommons.org/licenses/by-nc-nd/4.0/.

\section{References}

1. Rysavy MA, Li L, Bell EF, et al. Between-hospital variation in treatment and outcomes in extremely preterm infants. N Engl J Med 2015;372:1801-11.

2. Seaton SE, King S, Manktelow BN, et al. Babies born at the threshold of viability:changes in survival and workload over 20 years. Arch Dis Child Fetal Neonatal Ed 2013;98:F15-20.

3. Serenius F, Sjörs G, Blennow M, et al. EXPRESS study shows significant regional differences in 1-year outcome of extremely preterm infants in Sweden. Acta Paediatr 2014;103:27-37.

4. Costeloe KL, Hennessy EM, Haider S, et al. Short term outcomes after extreme preterm birth in England:comparison of two birth cohorts in 1995 and 2006 (the EPICure studies). BMJ 2012;345:e7976.

5. Hintz SR, Kendrick DE, Wilson-Costello DE, et al. Early-childhood neurodevelopmental outcomes are not improving for infants born at $<25$ weeks'gestational age. Pediatrics 2011;127:62-70.

6. Claas MJ, Bruinse HW, Koopman C, et al. Twoyear neurodevelopmental outcome of preterm born children $\leq 750 \mathrm{~g}$ at birth. Arch Dis Child Fetal Neonatal Ed 
2011; 96:F169-77.

7. Moore T, Hennessy EM, Myles J, et al. Neurological and developmental outcome in extremely preterm children born in England in 1995 and 2006: the EPICure studies. BMJ 2012;345:e7961.

8. Chalak LF, Tarumi T, Zhang R. The 'neurovascular unit approach' to evaluate mechanisms of dysfunctional autoregulation in asphyxiated newborns in the era of hypothermia therapy. Early Hum Dev 2014;90:687-94.

9. Shellhaas RA, Chang T, Tsuchida T, et al. The American Clinical Neurophysiology Society's Guideline on Continuous Electroencephalography Monitoring in Neonates. J Clin Neurophysiol 2011;28:611-7.

10. Azzopardi D, Strohm B, Edwards AD, et al. Treatment of asphyxiated newborns with moderate hypothermia in routine clinical practice:how cooling is managed in the UK outside a clinical trial. Arch Dis Child Fetal Neonatal Ed 2009;94:F260-4.

11. Toet MC, Lemmers PM. Brain monitoring in neonates. Early Hum Dev 2009;85:77-84.

12. Chen C, Sun C, Leonhardt S, et al. Amplitudeintegrated electroencephalography applications and algorithms in neonates: A systematic review. IEEE Access 2019;7:141766-81.

13. Rakshasbhuvankar A, Rao S, Ghosh S, et al. Why do neonates receive antiseizure medications? J Matern Fetal Neonatal Med 2020;14:1-5.

14. Perinatal Committee, Community of Pediatrics, Chinese Medical Association. Clinical application in neonatal amplitude-integrated electroencephalography-expert consensus. Chin J Neonatol 2019;34:3-7.

15. Boylan G, Burgoyne L, Moore C, et al. An international survey of EEG use in the neonatal intensive care unit. Acta

Cite this article as: Wang $\mathrm{Z}$, Zhang $\mathrm{P}$, Zhou W, Zhou X, Shi Y, Cheng X, Lin Z, Xia S, Zhou W, Cheng G. Electroencephalography monitoring in the neonatal intensive care unit: a Chinese perspective. Transl Pediatr 2021;10(3):552559. doi: 10.21037/tp-20-340
Paediatr 2010;99:1150-5.

16. Buttle SG, Sell E, Webster R, et al. Continuous electroencephalography monitoring for critically III neonates: A Canadian perspective. Can J Neurol Sci 2019;46:394-402.

17. Shah NA, Van Meurs KP, Davis AS. Amplitude-integrated electroencephalography: a survey of practices in the United States. Am J Perinatol 2015;32:755-60.

18. Ponnusamy V, Nath P, Bissett L, et al. Current availability of cerebral function monitoring and hypothermia therapy in UK neonatal units. Arch Dis Child Fetal Neonatal Ed 2010;95:F383-4.

19. Olischar M, Shany E, Aygün C, et al. Amplitude-integrated electroencephalography in newborns with inborn errors of metabolism. Neonatology 2012;102:203-11.

20. Olischar M, Klebermass K, Hengl B, et al. Cerebrospinal fluid drainage in posthaemorrhagic ventricular dilatation leads to improvement in amplitude-integrated electroencephalographic activity. Acta Paediatr 2009;98:1002-9.

21. Olischar M, Klebermass K, Kuhle S, et al. Progressive posthemorrhagic hydrocephalus leads to changes of amplitude-integrated EEG activity in preterm infants. Childs Nerv Syst 2004;20:41-5.

22. Hellström-Westas L, Klette H, Thorngren-Jerneck $\mathrm{K}$, et al. Early prediction of outcome with aEEG in preterm infants with large intraventricular hemorrhages. Neuropediatrics 2001;32:319-24.

23. Abend NS, Topjian AA, Gutierrez-Colina AM, et al. Impact of continuous EEG monitoring on clinical management in critically ill children. Neurocrit Care $2011 ; 15: 70-5$. 


\section{Supplementary}

\section{Appendix 1 Survey questions}

1. What is your primary centre of practice (Name of Institution, City)? (text box)

2. What is your specialty?
a. Neonatologist
b. Pediatrician (not neonatologist)
c. Nurse
d. Physiotherapist
e. Pharmacist
f. Neurodevelopmental evaluator
g. Inspector
h. Imaging physician
i. Other: (text box)

3. What is your professional title?
a. Senior
b. Intermediate
c. Junior
d. Other: (text box)

4. How many years do you work in your unit?
a. $0-5$
b. $6-10$
c. $11-15$
d. $16-20$
e. $>20$

5. In what type of hospital do you work?
a. General hospital
b. Maternity and infant hospital
c. Children's hospital
d. Other: (text box)

6. In what level of hospital do you work?
a. Grade III
b. Grade II
c. Grade I

7. How many beds in your neonatal intensive care unit?
a. $<20$
b. $20-50$
c. $51-70$
d. $71-100$
e. $>100$

8. Do you use any long term EEG monitoring (amplitudeintegrated or conventional EEG) in your NICU?
a. Yes
b. No (continue with 11 , then the survey is terminated)

9. If question 7 answered yes, which:
a. aEEG
b. cEEG
c. Combined aEEG/cEEG

10. How many EEG devices in your NICU?
a. $1-2$
b. $3-5$
c. $6-8$
d. $9-10$
e. $>10$

11. If you don't use any NICU EEG monitoring, why not? Please check all that apply.
a. EEG equipment is too expensive
b. Difficult to interpret
c. Other department has EEG capability
d. Other: (text box)

12. How many patients per day in your NICU would you monitor with long term EEG?
a. 1-2
b. $3-5$
c. $6-10$
d. $>10$

13. Who interprete the cEEG results in your unit?
a. Neonatologist
b. Pediatric neurologist
c. Other: (text box)

14. Who interprete the aEEG results in your unit?
a. Neonatologist
b. Pediatric neurologist
c. Other: (text box)

15. How long was EEG monitoring in neonates with brain injury?

a. $<2$ hour 

b. 2-5 hour
c. 6-12 hour
d. $>12$ hour
e. I am unsure

16. How long was EEG monitoring in neonates with seizures?
a. $<2$ hour
b. 2-5 hour
c. 6-12 hour
d. $>12$ hour
e. I am unsure

17. What is your preferred long term monitoring metod for assessing the following clinical scenarios for patients in the NICU?
a. Encephalopathy
b. Seizures/ suspected seizures
c. Neonates at high risk of brain injury
d. Assessment of brain development
e. All neonates admitted to the NICU

18. Do you currently manage patients based on bedside EEG monitoring?
a. Yes
b. No
c. I am unsure

19. Have you received any training in EEG use?
a. Yes
b. No

20. Have you read any professional books on EEG?
a. Yes
b. No

21. Would you like to attend an education session on EEG use in the NICU at your institution?
a. Yes
b. No
c. I am unsure 